\title{
A case report of $46, X Y$ partial gonadal dysgenesis caused by a novel mutation in the sex-determining region gene
}

\author{
Ke Xu, Na Su, Hong Zhang, Jingxin Zhu, Xinran Cheng \\ Department of Genetics and Endocrinology, Chengdu Women's and Children's Center Hospital, School of Medicine, University of Electronic \\ Science and Technology of China, Chengdu, China \\ Correspondence to: Xinran Cheng. Department of Genetics and Endocrinology, Chengdu Women's and Children's Center Hospital, School of \\ Medicine, University of Electronic Science and Technology of China, Chengdu, China. Email: cxr1216@sina.com.
}

\begin{abstract}
The sex-determining region Y (SRY) gene is a key gene involved in male sex differentiation and development. Patients with 46,XY disorders of sex development related to mutations in the high mobility group (HMG) box typically present with complete gonadal dysgenesis. In this study, we report a case of novel missense mutation c.T281G within the HMG domain of SRY in a 15-year-old patient of the female gender with 46,XY partial gonadal dysgenesis (PGD). The novel missense mutation caused the substitution of codon 94 for leucine in the HMG box of the SRY protein with an arginine codon. Leucine and arginine are aliphatic amino acids, and three-dimensional protein structure prediction revealed only slight structural changes in the SRY protein. Thus, the SRY protein had maintained some of its functions, and the patient presented with PGD. In conclusion, we identified a novel SRY mutation in a patient with 46,XY PGD. Based on the protein model, we believe that the mutation in the HMG domain helped to maintain the partial function of the SRY protein. The condition of our patient differed from the well-known 46,XY complete gonadal dysgenesis caused by mutations in the HMG region. In fact, this is the first case of 46,XY PGD caused by mutations in the HMG region to be reported, and therefore, our experience has expanded the mutation spectrum of the SRY gene. Furthermore, the present case demonstrates that mutations located in the HMG domain of SRY gene cannot be ruled out in patients with a clinical diagnosis of 46,XY PGD.
\end{abstract}

Keywords: 46,XY disorder of sex development (46,XY DSD); sex-determining region Y (SRY); partial gonadal dysgenesis (PGD)

Submitted Sep 16, 2020. Accepted for publication Dec 16, 2020.

doi: $10.21037 /$ tp-20-414

View this article at: http://dx.doi.org/10.21037/tp-20-414

\section{Introduction}

Disorders of sex development (DSDs) are genetically heterogeneous diseases involving inconsistencies in the chromosome karyotype, gonadal anatomy, and gonadal phenotype (1). The incidence of DSDs in live births is estimated to be in the range of $1 / 4,500$ to $1 / 5,000$ (2). Patients with DSDs present with diverse clinical manifestations. In some patients, genital ambiguity is apparent at birth, whereas others have typical male or female genitalia at birth but a different internal gonad structure, which can delay clinical diagnosis until adolescence or adulthood (3).

DSD with 46,XY chromosomal complement can be caused by different gene mutations during testicular differentiation, including mutations in SRY-box transcription factor 9 (SOX9), desert hedgehog signaling molecule (DHH), sex-determining region Y (SRY), nuclear receptor subfamily 5 group a member 1 (NR5A1), nuclear receptor subfamily 0 group b member 1 (NR0B1), Wilms' tumor suppressor gene 1 (WT1), doublesex- and mab3-related transcription factor 1 (DMRT1), and mitogenactivated protein kinase 1 (MAPK3K1).

SRY mutations are responsible for $10-15 \%$ of $46, \mathrm{XY}$ DSD cases (4). The SRY gene is considered to be a key gene in male sex differentiation and development. It encodes 204 amino acids and is a high-mobility group (HMG) box family 
member, which binds to DNA. The Y-encoded SRY gene is critical in the initiation of masculine differentiation of the gonads, establishing gonadal hormone differences between males and females, thus indirectly causing major sex differences in tissue function. SRY induces the development of relatively undifferentiated gonadal tissue into testicular tissue (5). The SRY protein contains a highly conserved DNA-binding domain, the HMG box, and on 2 sides of the HMG box are the less-conserved $\mathrm{N}$ - and C-terminal fragments. Point mutations, deletions, and translocations of the SRY gene can reduce the DNA-binding ability of SRY protein, leading to DSD. Most mutations are in the HMG box region, and 46,XY complete gonadal dysgenesis (46,XY CGD) is a consequence of such mutations. Meanwhile, mutations in the $\mathrm{N}$-terminus or $\mathrm{C}$-terminus of the SRY gene typically result in 46,XY partial gonadal dysgenesis (46,XY PGD). Here, we report a rare case of a patient with 46,XY PGD caused by mutations within the HMG domain of SRY.

We present the following article in accordance with the CARE reporting checklist (available at http://dx.doi. org/10.21037/tp-20-414).

\section{Case presentation}

A 15-year-old patient of the female gender sought treatment at our hospital for primary amenorrhea. The patient was born at full term via a normal delivery with a birth weight of $3.25 \mathrm{~kg}$. She had no family history of infertility, amenorrhea, abnormal development of external genitalia, or cryptorchidism. During physical examination, a height of $167.3 \mathrm{~cm}$, weight of $61 \mathrm{~kg}$, facial acne, and female external genitalia were observed; the patient's Tanner stage was B4PH4, and she had sparse armpit hair. Hormone testing revealed primary hypogonadism, with a follicle-stimulating hormone level of $40.11 \mathrm{mIU} / \mathrm{mL}$ (adult female follicular phase: $2.5-10.2 \mathrm{mIU} / \mathrm{mL}$ ), a luteinizing hormone level of $20.45 \mathrm{mIU} / \mathrm{mL}$ (adult female follicular phase: $1.9-12.5 \mathrm{mIU} / \mathrm{mL}$ ), an anti-mullerian hormone level of $0.79 \mathrm{ng} / \mathrm{mL}$, an estradiol level of $42.86 \mathrm{pg} / \mathrm{mL}$ (reference value: $19.5-356.7 \mathrm{pg} / \mathrm{mL}$ ), and a testosterone level of $127.92 \mathrm{ng} / \mathrm{dL}$ (reference value $<38.92 \mathrm{ng} / \mathrm{dL}$ ). The levels of $17 \alpha$-hydroxyprogesterone, adrenocorticotropic hormone, and dehydroepiandrosterone sulfate were in the normal range. Therefore, adrenal hyperandrogenism could be excluded. The patient had normal prolactin levels and adrenal gland examination results. The patient's karyotype was $46, \mathrm{XY}$, with no chimera. Ultrasound examination showed the uterus to be approximately $2.0 \mathrm{~cm} \times 1.6 \mathrm{~cm} \times$ $1.5 \mathrm{~cm}$ in size, an endometrial thickness of approximately $0.22 \mathrm{~cm}$, and the left and right ovaries to measure $1.9 \mathrm{~cm} \times 1.0 \mathrm{~cm}$ and $2.2 \mathrm{~cm} \times 0.9 \mathrm{~cm}$, respectively. No obvious follicles were observed in either ovary, which was suggestive of an infantile uterus. Pelvic magnetic resonance imaging indicated gonadal dysgenesis, an anteverted uterus, an approximate ovary size of $14.5 \mathrm{~mm} \times 33 \mathrm{~mm}$, an approximate cervical size of $10 \mathrm{~mm} \times 21 \mathrm{~mm}$, and an endometrial thickness of $3 \mathrm{~mm}$. The appearance of the vagina on magnetic resonance imaging was discontinuous; the middle and upper vaginal canal had a cord-like shape, and no lumen was apparent. The lower vaginal wall was clearly visible. A soft oval tissue signal was observed near the right pelvic wall, with dot-like high signal intensity on $\mathrm{T}_{2}$-weighted imaging, and the signal was approximately $21 \mathrm{~mm} \times 13 \mathrm{~mm}$ in size. The patient had a bone age of 16 years. Gonad biopsy revealed that the upper, middle and lower poles of the left gonad consisted of ovarian tissue, mainly ovarian interstitial tissue, with no follicular structure. In the interstitial tissue, lymphocytic infiltration accompanied by calcification was observed. At the upper pole of the right gonad, gonadal dysgenesis with gonadoblastoma was observed along with extensive calcification; however, no interstitial infiltration was visible. At the lower pole of the right gonad, gonadal dysgenesis with extensive calcification was observed, with no tumors.

\section{Research methods}

The protocol was approved by the medical ethics committee, and informed consent was obtained from the patient's parents. All procedures performed in studies involving human participants were in accordance with the Helsinki Declaration (as revised in 2013). First, a DNA extraction kit (Tiangen Biotech Beijing Co., Ltd., Beijing, China) was used to extract DNA from peripheral blood samples from the patient and her parents. After DNA quality verification, 281 candidate genes known to be related to DSDs were captured using a target sequence capture chip (MyGenostics, Beijing, China). The sequences were obtained using the sequencing platform of an Illumina HiSeq 2000 sequencer (Illumina, San Diego, CA, USA). The original sequencing data were filtered, and then the selected sequences were aligned to the Homo sapiens (human) genome assembly GRCh37 (hg19) in the NCBI database using BurrowsWheeler Aligner (BWA, http://biobwa.sourceforge.net/) software. GATK (https://software.broadinstitute.org/gatk/) 


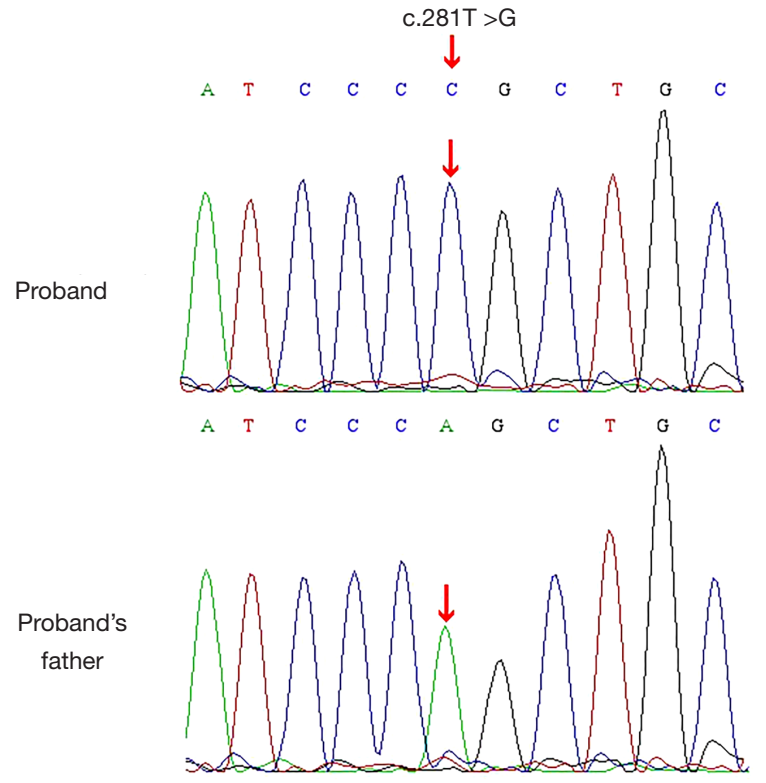

Figure 1 SRY gene sequencing validation. The SRY gene was transcribed and translated using the antisense strand as a template. The arrow indicates the mutation site. The patient has a novel mutation in the SRY gene c.281T $>\mathrm{G}$, but her father has no such mutation.

was used to analyze the data of single nucleotide variations $(\mathrm{SNVs})$, and insertions and deletions (INDELs). Then, all single-nucleotide polymorphisms (SNPs) and INDELs were annotated in ANNOVAR (http://annovar.openbioinformatics. org/en/latest/). Mutation sites with frequencies $<0.02$ in the normal human database were excluded. PolyPhen-2 (http://genetics.bwh.harvard.edu/pph2/), MutationTaster (http://www.mutationtaster.org/), and GERP ++ (http://mendel.stanford.edu/SidowLab/downloads/gerp/ index.html) were used for the prediction of pathogenicity and conservation of missense mutations (http://sift.jcvi.org/). The selected mutation sites were verified by polymerase chain reaction and Sanger sequencing. Co-segregation validation of verified variants was performed for the family members. According to the standards and guidelines of the American College of Medical Genetics (ACMG), the mutation was classified as a pathogenic mutation.

The results of gene detection (Figure 1) showed that the thymidine at codon 281 of the SRY gene of the patient had mutated to guanine (i.e., c.281T $>\mathrm{G}$ ). This mutation had led to the substitution of codon 94 for leucine in the HMG box of the SRY protein with the codon for arginine (p. L94R). The mutation could not be found in the Human Gene Mutation Database (HGMD). The patient's father had a normal SRY gene, with no corresponding mutation, suggesting that the patient's mutation was a novel mutation that had occurred during embryonic development. The results of the pathological examination of the patient's gonads are shown in Figure 2.

\section{Discussion}

Human sex determination is a complex process that is jointly affected by genetic and epigenetic factors (6). Current data show that genetic testing to identify the cause of the disease can only be performed in $43 \%$ of $46, \mathrm{XY}$ DSD cases (7). The SRY gene is the most commonly mutated gene in patients with 46,XY DSD (8). To date, more than 100 sexual development abnormalities associated with mutations in the open reading frame or noncoding region of the SRY gene have been reported on the HGMD database. The SRY protein contains a highly conserved DNA-binding domain, the HMG box, and 2 sides of the HMG box in SRY are the less-conserved $\mathrm{N}$ - and C-terminal fragments (9). Through the HMG box, SRY interacts with specific DNA sequences to activate the transcription of the testicular determinant gene SOX9, thus playing a critical role as a transcription factor in sex determination (10). Most mutations are in the HMG box region (11). Mutations in this region often result in 46,XY CGD. Due to the lack of testicular tissue, patients with 46,XY CGD are unable to secrete androgen and antimullerian hormone; as a result, their reproductive systems 

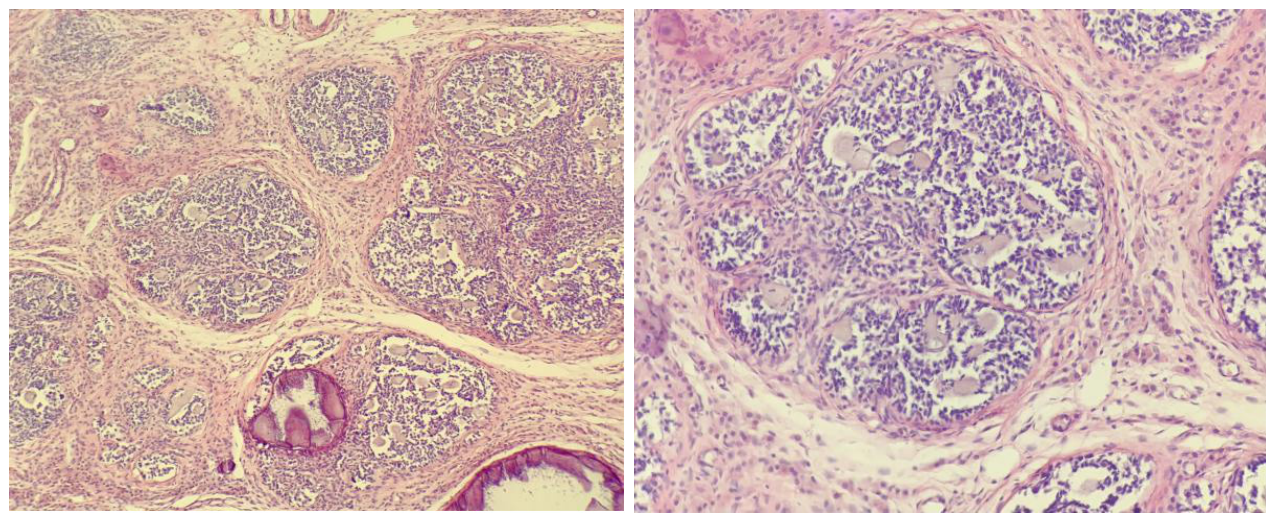

Figure 2 Pathological findings of the gonads (Hematoxylin-Eosin Staining). Under a microscope (left, HE, x40; right HE, x100), round nests of cancer cells are visible (mainly sex cord-like components and primordial germ cells). The cancer cell nests form the small acinus surrounding the primordial germ cells, with calcification around them, which is suggestive of gonadal dysgenesis with gonadoblastoma and extensive calcification (upper pole of the right gonad).

differentiate into female reproductive systems internally and externally. Due to gonadal dysgenesis and ovarian dysfunction, patients with 46,XY CGD are often diagnosed due to primary amenorrhea at puberty. Mutations in the $\mathrm{N}$-terminus or C-terminus of the SRY gene often result in 46,XY PGD. The gonads of patients with this DSD are usually a mixture of Wolffian and Mullerian ducts, and the unilateral or bilateral gonads may have the ability to produce sex hormones, albeit not enough to reach normal levels. The degree of masculinization and the degree of Mullerian duct regression depend on testicular function.

In this study, we discovered a novel missense mutation related to 46,XY PGD that has not been reported in the HGMD. The L94R mutation is located in the highly conserved HMG box region of the SRY gene. Sex hormone examination of our patient revealed hypogonadotropic hypogonadism accompanied by hyperandrogenism. The patient had normal levels of $17 \alpha$-hydroxyprogesterone, adrenocorticotropic hormone, and dehydroepiandrosterone sulfate, which excluded adrenal hyperandrogenism. Furthermore, gonadal biopsy did not detect any androgensecreting tumors, which is consistent with PGD. Mitchell et al. studied the effects of different amino acid substitutions at the same locus (L94P) on the DNA-binding and -bending functions of SRY. Studies have shown that the L94P mutation can break the structure of the second $\alpha$-helix of the HMG domain and reduce its binding affinity to DNA to undetectable levels, which severely interferes with DNAbinding activity, resulting in CGD (12). The finding of the present case was inconsistent with the previous findings that mutations in the HMG box domain usually lead to 46,XY CGD. The possible reasons for this are as follows. Firstly, the L94R mutation leads to the replacement of leucine with arginine, both of which are aliphatic amino acids, with no obvious changes in the degree of DNA binding revealed by three-dimensional prediction. This indicates that the mutation in our patient caused minor structural changes in the SRY protein, which had a relatively small effect on its affinity for DNA binding. Therefore, the residual SRY protein led to PGD. Secondly, the phenotype of any single mutation may be related to a patient's genetic background or certain environmental factors. It has been reported that a mutation in the HMG domain was found in the father of a female with 46,XY (the father was non-mosaicism), suggesting that the genetic background may change or compensate for the effects of mutations in nonaffected carriers.

The main differential diagnosis of PGD is mixed gonadal dysgenesis (MGD), which is one of the DSDs associated with sex chromosome abnormalities (13). PGD patients and MGD patients possess similar gonadal and external genitalia characteristics; however, MGD patients also have a $45 \mathrm{X}$ cell line and one or more chimeras with a normal or abnormal Y lineage structure. Therefore, MGD patients may present with clinical manifestations of Turner syndrome, including short stature, deformity, and cardiovascular and renal malformations (14).

Patients with 46,XY DSD carry an extremely high risk of developing germ-cell tumors, which is in the range of $30 \%$ to $75 \%)(15-17)$ and increases with age. Gonadoblastoma is 
the most common precursor of germ-cell malignancies in individuals with DSD and often affects the right gonad. The gonadoblastoma susceptibility region is localized in a small region near the centromere of the $\mathrm{Y}$ chromosome. Studies have demonstrated that genes such as testis-specific protein, Y-encoded and SRY in the gonadoblastoma susceptibility region are associated with gonadoblastoma and dysgerminomas. Therefore, accurate molecular diagnosis is crucial for the management of 46,XY DSD patients as well as for predicting the risk of malignant tumors. Therefore, considering the high risk of malignant germ-cell tumors, following our patient's diagnosis, we immediately removed the affected side to prevent the occurrence of a gonadal malignancy. Some studies have also suggested that due to the possible existence of gonadal chimera in parents, sisters of clinical probands should also be screened for SRY mutations in order to prevent sex hormone secretion disorders and the development of latent gonadal tumors (18).

While gonadal diseases are rarely fatal, they do affect the quality of life of patients. Since these diseases have a high degree of clinical phenotypic heterogeneity, the treatment must be individualized and tailored. Individualized treatment protocols that are feasible for pediatric patients and their families should be established with consideration given to the clinical phenotype, sexual orientation, familial and social acceptance, and other factors. First and foremost, potentially life-threatening factors must be promptly eliminated. In the current case, gonadal histopathology revealed the presence of GD with rightsided adenoblastoma, which might have had the potential to transform into a germ-cell malignancy such as embryonic carcinoma, yolk sac tumor, immature teratoma, or choriocarcinoma (19). Therefore, the gonad on the affected side was removed immediately after diagnosis to prevent the development of a malignant gonadal tumor. Second, the child was raised as a female, both psychologically and socially. After resection of the right gonad, a female identity was socially maintained for the child. Thus, hormone replacement therapy was particularly important for her in order to induce puberty, simulate artificial cycles, and prevent osteoporosis. The existence of the $\mathrm{Y}$ chromosome does not affect the effects of drug treatment in assisted reproductive technology or hormones during pregnancy on the endometrium. Many cases have been reported of 46,XY CGD patients becoming pregnant and successfully giving birth through IVF with donor eggs after the development of the uterus to a normal size. Although our current case was a pediatric patient with 46,XY PGD, hyperandrogenemia was no longer detected during follow-up visits after the removal of the right gonad. We believe that our patient will be able to become pregnant and enjoy a normal life after systematic treatment and subsequent adjustment of the treatment plan during the long-term follow-up. To sum up, the health consequences of DSD are manifold and can include sex hormone disorders, impaired fertility, an increased risk of gonadal tumors, and gender dysphoria/psychosexual disorders caused by social stigma. Therefore, successful management of DSD is extremely complicated and requires a multidisciplinary approach.

In conclusion, we discovered a novel pathogenic mutation that caused 46,XY PGD, namely, c.281T>G (p.L94R). Our experience provides evidence that as well as mutations in the less-conserved $\mathrm{N}$ - and $\mathrm{C}$-terminal fragments, mutations in the HMG box region of the SRY gene can also lead to 46,XY PGD. Therefore, our findings expand the pathogenic mutation spectrum of 46,XY gonadal dysgenesis and improve the etiological diagnostic level of 46,XY DSD. However, in the majority of DSD cases, the genetic etiology is unknown, which shows that our knowledge of the genetic pathways involved in DSDs is lacking. High-throughput sequencing will assist in revealing the rare gene mutations that cause these phenotypes. However, to completely determine the causal relationship of these mutations and to better understand their mechanism, a series of studies using different experimental models needs to be conducted.

\section{Acknowledgments}

Funding: This study was supported by Sichuan Science and Technology Project (2019JDPT0034).

\section{Footnote}

Reporting Checklist: The authors have completed the CARE reporting checklist. Available at http://dx.doi.org/10.21037/ tp-20-414

Conflicts of Interest: All authors have completed the ICMJE uniform disclosure form (available at http://dx.doi. org/10.21037/tp-20-414). The authors have no conflicts of interest to declare.

Ethical Statement: The authors are accountable for all aspects of the work in ensuring that questions related to the accuracy or integrity of any part of the work are appropriately investigated and resolved. The protocol was 
approved by the medical ethics committee, and parental informed consent was received. All procedures performed in studies involving human participants were in accordance with the Helsinki Declaration (as revised in 2013).

Open Access Statement: This is an Open Access article distributed in accordance with the Creative Commons Attribution-NonCommercial-NoDerivs 4.0 International License (CC BY-NC-ND 4.0), which permits the noncommercial replication and distribution of the article with the strict proviso that no changes or edits are made and the original work is properly cited (including links to both the formal publication through the relevant DOI and the license). See: https://creativecommons.org/licenses/by-nc-nd/4.0/.

\section{References}

1. Lee PA, Houk CP, Ahmed SF, et al. Consensus statement on management of intersex disorders. International Consensus Conference on Intersex. Pediatrics 2006;118:e488-500.

2. Warne GL, Raza J. Disorders of sex development (DSDs), their presentation and management in different cultures. Rev Endocr Metab Disord 2008;9:227-36.

3. Kutney K, Konczal L, Kaminski B, et al. Challenges in the diagnosis and management of disorders of sex development. Birth Defects Res C Embryo Today 2016;108:293-308.

4. Andonova S, Robeva R, Sirakov M, et al. A Novel SRY Gene Mutation p.F109L in a 46,XY Female with Complete Gonadal Dysgenesis. Sex Dev 2015;9:333-7.

5. Koopman P. The delicate balance between male and female sex determining pathways: potential for disruption of early steps in sexual development. Int J Androl 2010;33:252-8.

6. Gunes SO, Metin Mahmutoglu A, Agarwal A. Genetic and epigenetic effects in sex determination. Birth Defects Res C Embryo Today 2016;108:321-36.

7. Eggers S, Sadedin S, van den Bergen JA, et al. Disorders of sex development: insights from targeted gene sequencing of a large international patient cohort. Genome Biol 2016;17:243.

Cite this article as: $\mathrm{Xu} \mathrm{K}$, Su N, Zhang $\mathrm{H}$, Zhu J, Cheng X. A case report of $46, X Y$ partial gonadal dysgenesis caused by a novel mutation in the sex-determining region gene. Transl Pediatr 2020;9(6):867-872. doi: 10.21037/tp-20-414
8. Bashamboo A, McElreavey K. Mechanism of Sex Determination in Humans: Insights from Disorders of Sex Development. Sex Dev 2016;10:313-25.

9. Sinclair AH, Berta P, Palmer MS, et al. A gene from the human sex-determining region encodes a protein with homology to a conserved DNA-binding motif. Nature 1990;346:240-4.

10. Capel B. Vertebrate sex determination: evolutionary plasticity of a fundamental switch. Nat Rev Genet 2017;18:675-89.

11. Helszer Z, Dmochowska A, Szemraj J, et al. A novel mutation (c. $341 \mathrm{~A}>\mathrm{G}$ ) in the SRY gene in a $46, \mathrm{XY}$ female patient with gonadal dysgenesis. Gene 2013;526:467-70.

12. Mitchell CL, Harley VR. Biochemical defects in eight SRY missense mutations causing XY gonadal dysgenesis. Mol Genet Metab 2002;77:217-25.

13. Hughes IA, Houk C, Ahmed SF, et al. Consensus statement on management of intersex disorders. Arch Dis Child 2006;91:554-63.

14. Gabriel Ribeiro de Andrade J, Marques-de-Faria AP, Fabbri HC, et al. Long-Term Follow-Up of Patients with 46,XY Partial Gonadal Dysgenesis Reared as Males. Int J Endocrinol 2014;2014:480724.

15. Michala L, Goswami D, Creighton SM, et al. Swyer syndrome: presentation and outcomes. BJOG 2008;115:737-41.

16. Zieliñska D, Zajaczek S, Rzepka-Górska I. Tumors of dysgenetic gonads in Swyer syndrome. J Pediatr Surg 2007;42:1721-4.

17. Capito C, Leclair MD, Arnaud A, et al. 46,XY pure gonadal dysgenesis: clinical presentations and management of the tumor risk. J Pediatr Urol 2011;7:72-5.

18. Stoppa-Vaucher S, Ayabe T, Paquette J, et al. 46, XY gonadal dysgenesis: new SRY point mutation in two siblings with paternal germ line mosaicism. Clin Genet 2012;82:505-13.

19. Simon RA, Laughlin TS, Nuccie B, et al. A $46 \mathrm{XY}$ phenotypic female adolescent with bilateral gonadal tumors consisting of five different components. Int J Gynecol Pathol 2008;27:407-11. 